\title{
Sub-fs electron bunch generation with sub-10-fs bunch arrival-time jitter via bunch slicing in a magnetic chicane
}

\author{
J. Zhu, ${ }^{1,2,{ }^{*}}$ R. W. Assmann, ${ }^{1}$ M. Dohlus, ${ }^{1}$ U. Dorda, ${ }^{1}$ and B. Marchetti ${ }^{1}$ \\ ${ }^{1}$ Deutsches Elektronen-Synchrotron, DESY, Hamburg, Germany \\ ${ }^{2}$ Universität Hamburg, Hamburg, Germany
}

(Received 31 August 2015; revised manuscript received 5 January 2016; published 19 May 2016)

\begin{abstract}
The generation of ultrashort electron bunches with ultrasmall bunch arrival-time jitter is of vital importance for laser-plasma wakefield acceleration with external injection. We study the production of $100-\mathrm{MeV}$ electron bunches with bunch durations of subfemtosecond (fs) and bunch arrival-time jitters of less than $10 \mathrm{fs}$, in an S-band photoinjector by using a weak magnetic chicane with a slit collimator. The beam dynamics inside the chicane is simulated by using two codes with different self-force models. The first code separates the self-force into a three-dimensional (3D) quasistatic space-charge model and a one-dimensional coherent synchrotron radiation (CSR) model, while the other one starts from the first principle with a so-called 3D sub-bunch method. The simulations indicate that the CSR effect dominates the horizontal emittance growth and the 1D CSR model underestimates the final bunch duration and emittance because of the very large transverse-to-longitudinal aspect ratio of the sub-fs bunch. Particularly, the CSR effect is also strongly affected by the vertical bunch size. Due to the coupling between the horizontal and longitudinal phase spaces, the bunch duration at the entrance of the last dipole magnet of the chicane is still significantly longer than that at the exit of the chicane, which considerably mitigates the impact of space charge and CSR effects on the beam quality. Exploiting this effect, a bunch charge of up to $4.8 \mathrm{pC}$ in a sub-fs bunch could be simulated. In addition, we analytically and numerically investigate the impact of different jitter sources on the bunch arrival-time jitter downstream of the chicane, and define the tolerance budgets assuming realistic values of the stability of the linac for different bunch charges and compression schemes.
\end{abstract}

DOI: 10.1103/PhysRevAccelBeams.19.054401

\section{INTRODUCTION}

Laser-plasma wakefield acceleration (LWFA) is an attractive and promising technology for very compact accelerators and light sources as the plasma wave can sustain accelerating gradients several orders of magnitude higher than conventional accelerators [1-4]. There are already many running and planned experiments in laboratories worldwide aiming to develop such a novel technology. Internal injection and external injection of electrons are the two schemes for highbrightness electron sources in LWFA. External injection allows the precise manipulation of the phase space of the incoming electron bunches. It therefore provides the possibility to optimize the beam dynamics inside the plasma.

In order to achieve high-quality electron bunches by LWFA with external injection, the generation of ultrashort and precisely synchronized electron bunches, e.g. by conventional accelerators, is of vital importance. In a plasma accelerator, the plasma wavelength is inversely proportional

\footnotetext{
jun.zhu@desy.de

Published by the American Physical Society under the terms of the Creative Commons Attribution 3.0 License. Further distribution of this work must maintain attribution to the author $(s)$ and the published article's title, journal citation, and DOI.
}

to the acceleration gradient. Assuming for example a plasma density of $10^{17} \mathrm{~cm}^{-3}$, the acceleration gradient is approximately $30 \mathrm{GV} / \mathrm{m}$ and the plasma wavelength is around $100 \mu \mathrm{m}$. Therefore, an ultrashort electron bunch is required to reduce the final energy spread and a precise synchronization between the driving laser and the electron is needed to reduce the shot-to-shot energy variation [5].

Plasma wakefield acceleration (PWFA) utilizes the plasma wave excited by an electron bunch or bunch train to accelerate the trailing witness bunch [6]. The length of the witness bunch in PWFA has similar requirements like that for LWFA with external injection.

More generally, ultrashort electron bunches produced in conventional or advanced accelerators can be used to generate pulses of light with sub-fs duration, which are of great interest due to their ability to probe electronic dynamics on the atomic scale [7-8]. For example, the rms pulse length for single-spike self-amplified spontaneous emission (SASE) x-ray free-electron laser (FEL) by using an electron beam of $2 \mathrm{GeV}$ is on the order of $1.5 \mathrm{fs}(1 \mathrm{pC})$ [9].

In this paper, we present theoretical and numerical studies of sub-fs electron bunch generation at $\sim 100-\mathrm{MeV}$ with a bunch charge of up to several $\mathrm{pC}$ using normal conducting $S$-band accelerator technologies as foreseen to be installed in the ARES-linac [10] at the upcoming accelerator R\&D 
facility SINBAD (short innovative bunches and accelerators at DESY) [11]. After acceleration, the electron bunch is compressed by a magnetic chicane with a slit (collimator) located between the second and the third dipole magnet. The maximum charge of the sub-fs bunch achieved in our study is 1 order of magnitude higher than simulation results obtained by using other compression schemes [12-13]. At the same time, the simulated transverse emittance is still sufficient for most of the envisaged applications. We also present the simulation results obtained by using a hybrid compression scheme, in which velocity bunching [14] is implemented prior to the magnetic compression. Furthermore, the influence of the bunch duration at the entrance of the chicane on the final bunch duration is discussed in detail. Finally, the bunch arrival-time jitter (timing jitter) of the sub-fs bunch downstream of the chicane is studied both analytically and numerically. The tolerances are found to be different to the more common case with bunches being eventually compressed without the slit. We show that a timing jitter of less than $10 \mathrm{fs}$ can be achieved assuming realistic values for the stability of the linac.

Another major novelty of this study lies in the depth of understanding compressing electron bunches to sub-fs durations when the space charge and coherent synchrotron radiation (CSR) effects are both non-negligible. The impact of the CSR effect on the beam quality during the bunch compression, when the space-charge effects are negligible, has been investigated comprehensively during the past thirty years [15-20]. In recent years, there has been increasing interest in compressing electron bunches at low energy, where the space-charge effects are still strong enough to spoil the beam quality [21-24]. There are generally two types of models (codes) to simulate the space-charge and CSR effects simultaneously. The first model separates them in a three-dimensional (3D) quasistatic space-charge model and a one-dimensional (1D) CSR model, e.g. PARMELA_B [25], IMPACT-T [26-27] and IMPACT-Z [28]. As this allows for fast computations it is thus adopted in most of the reported studies. In principle, however, the space-charge and CSR forces cannot be separated as they originate from the same Liénard-Wiechert (L-W) potential, and the 1D CSR approximation only works well under certain conditions [29]. The second model starts from the first-principle calculation, e.g. CSRTrack [30], Trafic4 [31], Tredi [32] and the L-W particle-mesh code reported by Ryne [33]. However, simulations with these codes are significantly more time consuming. Prokop et al. [21] compared the simulation results from IMPACT-Z and CSRTrack for the full compression of $\sim 40-\mathrm{MeV}$ electron bunches with bunch charges and durations down to $20 \mathrm{pC}$ and 40 fs respectively. While it was found that the emittance growth in the bending plane simulated by the latter is much higher than by the former, the reason was not found. In order to better understand magnetic bunch compression with non-negligible space-charge effects, especially when the final bunch duration is shorter than $1 \mathrm{fs}$, we present detailed beam dynamics studies using IMPACT-T and CSRTrack in order to understand the difference between the results from the two self-force models.

\section{TRADE-OFF OF PARAMETERS}

In this paper, the coordinates of an electron in $6 \mathrm{D}$ phase space is denoted as $\left[x, x^{\prime}, y, y^{\prime}, z, \delta\right]$. Here $z$ is the longitudinal distance from the center of the electron bunch and $\delta$ denotes the fractional energy spread. In the phase space plot of the electron beam, we use $t=z / c$ as the longitudinal coordinates and the convention that the bunch head is on the left side. Here $c$ denotes the velocity of light.

The schematic of the main beam line of the SINBAD linac is shown in Fig. 1. The $\sim 5-\mathrm{MeV}$ electron bunches generated by the 1.5-cell S-band photocathode rf gun are accelerated off-crest to approximately $100 \mathrm{MeV}$ by two 5.2-m-long S-band traveling-wave structures, which are powered by two independent klystrons. We keep the option to add a third traveling-wave structure in the future but it is not included in this study. Downstream of the travelingwave structures the main beam line includes quadrupole magnets and a magnetic chicane bunch compressor. A slit will be installed in the middle of the chicane to allow only the central slice of a ps or sub-ps bunch to pass through. This technique was first proposed by Borland [34] to generate 10-20 fs electron bunches at the Advanced Photo Source (APS) Linac. Recently, it has been demonstrated that this technique can be used for ultrashort FEL and electron bunch generation [35], beam diagnostics [36], as well as removing a double-horn structure in the current profile to improve FEL performance [37].

In this paper, we only consider the case of full compression of the initial bunch. Therefore, the chirp of the bunch at the entrance of the chicane is approximately

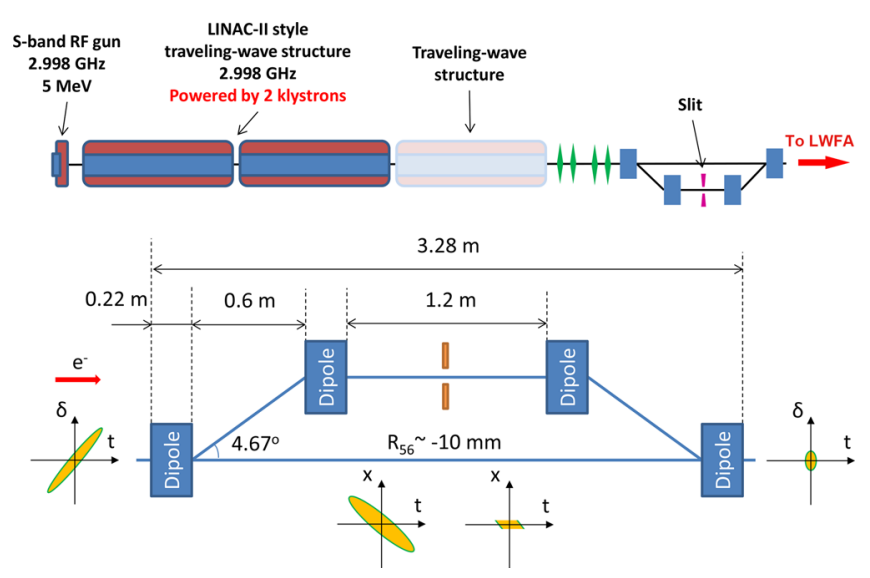

FIG. 1. Layout of the SINBAD linac (up) and schematic of the magnetic chicane with a slit (down). The blue rectangles represent dipole magnets while the green diamonds are quadrupole magnets. 
$h \approx-1 / R_{56}$, where $R_{56}$ is the longitudinal dispersion of the chicane. Here the chirp is defined as $h=\langle\delta z\rangle /\left\langle z^{2}\right\rangle$. At the SINBAD linac, the nominal $R_{56}$ of the chicane is $-10 \mathrm{~mm}$, which was identified as the optimal trade-off of the constraints discussed below.

Neglecting the high-order effect and the collective effects, at maximum compression the bunch length achievable by a chicane is given by

$$
\sigma_{z_{f}} \approx\left|R_{56}\right| \sigma_{\delta_{u}},
$$

where $\delta_{u}$ is the initial uncorrelated energy spread of the bunch. Equation (1) indicates that $R_{56}$ should be as weak as possible in order to obtain sub-fs bunches. For instance, the bunch duration given by Eq. (1) is $1.0 \mathrm{fs}$ for $R_{56}=-30 \mathrm{~mm}$ and $\delta_{u}=10^{-5}(1 \mathrm{keV}$ for a $100-\mathrm{MeV}$ bunch). Furthermore, during the compression of the electron bunch in a magnetic chicane, the energy spread of the bunch will increase due to the CSR effect, which in turn will result in a longer final bunch length. The CSR effect can be reduced by using a weak chicane and a large initial correlated energy spread.

Moreover, the contributions from the rf amplitude jitter of the traveling-wave structure and the magnetic field jitter of the dipole magnet to the rms timing jitter both scale as $R_{56} / c$. For example, a rf amplitude jitter or a magnetic field jitter of $0.01 \%$ corresponds to a contribution of 10.0 fs for $R_{56}=-30 \mathrm{~mm}$. Therefore, a weak $R_{56}$ is strongly desired.

However, on the other hand, reducing the $R_{56}$ also reduces the overall achieved energy gain for a given $\mathrm{rf}$ power. A maximum gradient of $24 \mathrm{MV} / \mathrm{m}$ is required for two 5.2-m-long traveling-wave structures, which are operated at the same gradient and phase, to accelerate electrons to $100-\mathrm{MeV}$ with a chirp of $100 \mathrm{~m}^{-1}\left(R_{56}=-10 \mathrm{~mm}\right)$.

For the LWFA experiment, the SINBAD linac must be able to operate at several working points with different bunch charges. The simplest method to tune the final bunch charge is changing the slit width. However, for the sub-fs bunch generation, the nonlinearity of the longitudinal phase space and the high-order longitudinal dispersion terms of the bunch compressor dominate the final bunch length. The current profile associated with the fully compressed bunch generally consists of a high peak in the bunch head with a long trailing tail. Therefore, the final bunch length (the tail of the bunch) increases quickly as the slit width increases.

There is also the possibility to employ a longitudinal phase space linearizer [38] before bunch compression. Then a slit with larger aperture can be used to slice more electrons from the initial bunch. However, this method requires additional, costly hardware and intrinsically implies an increase of the final energy spread of the beam, which will result in significant emittance growth due to chromatic aberration [39] in the matching section and spot size dilution at the entrance of the plasma.
To first order, the chromatic dilution of the beam size at the focal point is given by [40]

$$
\frac{\Delta \sigma_{\perp}}{\sigma_{\perp}} \approx \sigma_{\delta} \frac{L}{\beta_{\perp}},
$$

where $\sigma_{\perp}$ is the rms transverse beam size, $\sigma_{\delta}$ is the rms energy spread, $L$ is the focal length and $\beta_{\perp}$ is the beta function at the focal point. At the entrance of the chicane, the rms energy spread $\sigma_{\delta}$ of the bunch with length $\sigma_{z_{0}}$ and linear longitudinal phase space is approximately $\sigma_{\delta} \approx \sigma_{z_{0}} /\left|R_{56}\right|$. Assuming $R_{56}=-10 \mathrm{~mm}$, $\sigma_{z_{0}}=0.1 \mathrm{~mm}, \beta_{\perp}=1 \mathrm{~mm}$ and $L=0.3 \mathrm{~m}$, we have $\sigma_{\delta}=0.01$ and $\Delta \sigma_{\perp} / \sigma_{\perp}=3$.

Instead of using a linearizer, the bunch charge extracted from the photocathode can be adjusted while keeping the slit width sufficiently small. The latter method was adopted in our studies.

\section{METHODOLOGY OF SIMULATION MODELS}

The start-to-end (S2E) simulation of the beam dynamics in the SINBAD linac was performed with a combination of different codes. The electron bunch was first transported to the end of the linac by using ASTRA with a twodimensional cylindrical-symmetric space-charge algorithm [41] and four million macroparticles. The photocathode laser pulse was assumed to have a Gaussian longitudinal shape with an rms duration of $3 \mathrm{ps,}$, and a uniform transverse laser intensity distribution was taken at the photocathode. An initial kinetic energy of $0.75 \mathrm{eV}$ was used to simulate the thermal emittance contribution due to photoemission from the cathode. The two traveling-wave structures are operated at the same gradient and phase (offcrest) in order to minimize the timing jitter [42]. Therefore the electron bunch will be slightly compressed by velocity bunching before the chicane.

Downstream of the traveling-wave structures, the beam was tracked up to the end of the last dipole magnet by using IMPACT-T and CSRTrack respectively. In IMPACT-T, a 3D quasistatic space-charge algorithm and 1D CSR model were used, and the real on-axis magnetic field map of the dipole magnet was included. We also switched off the CSR in the IMPACT-T simulations to observe the impact from the space-charge effects alone. There are two different types of CSR models in CSRTrack. One is the 1D CSR model, and the other is the so-called "sub-bunch" model, in which the beam is tracked by the following procedure in each time step: (i) First, the 3D distributed particles are projected onto the bending plane and the retarded trajectories are calculated by back-tracking without the inclusion of the self-force. (ii) Second, the "point-to-point" L-W potentials are evaluated by replacing each source particle with a 3D Gaussian sub-bunch having equal charge and shape, i.e. the "sub-bunch-to-point" interaction is calculated with all the "points" on the bending plane. Since the center of 
each sub-bunch is the location of the corresponding source particle, the self-force has no vertical component because of the symmetry of the bending plane. (iii) Finally, the initial 3D distributed particles are pushed by the 2D selfforce and 3D external forces (e.g. the magnetic field of the dipole magnet). A hard-edge model of the dipole magnet was included in CSRTrack.

In CSRTrack, the 3D integration of the retarded source is calculated by the convolution of the quantities of a line charge density $\lambda(s, t)$ traveling along the path $\boldsymbol{r}_{s}(s)$ with a transverse density function $\eta(x, y)$ [43],

$$
\boldsymbol{X}(\boldsymbol{r}, t)=\int \boldsymbol{X}^{(\lambda)}\left(\boldsymbol{r}-\boldsymbol{r}_{\eta}(x, y), t\right) \eta(x, y) d x d y,
$$

where $\boldsymbol{X}$ and $\boldsymbol{X}^{(\lambda)}$ are the 3D and 1D quantities respectively. In the Lorentz gauge, the 1D scalar and vector potential are given by

$$
\begin{gathered}
\Phi^{(\lambda)}(\boldsymbol{r}, t)=\frac{1}{4 \pi \varepsilon_{0}} \int \frac{\lambda\left(s, t^{\prime}\right)}{R(\boldsymbol{r}, s)} d s, \\
\mathbf{A}^{(\lambda)}(\boldsymbol{r}, t)=\frac{\beta}{4 \pi \varepsilon_{0} c} \int \frac{\lambda\left(s, t^{\prime}\right)}{R(\boldsymbol{r}, s)} \boldsymbol{u}_{s}(s) d s,
\end{gathered}
$$

where $\varepsilon_{0}$ is the permittivity of free space, $\beta$ is the particle's velocity divided by the speed of light, $R(\boldsymbol{r}, s)=\boldsymbol{r}-\boldsymbol{r}_{s}$, $t^{\prime}=t-R(\boldsymbol{r}, s) / c$ and $\boldsymbol{u}_{s}(s)$ is the unity vector in the tangential direction of the trajectory. Since the bending plane is the symmetry plane for all the sub-bunches, the vertical sub-bunch size determines the volume of the 3D charge distribution during the self-force calculation, while the longitudinal and horizontal sub-bunch sizes determine the resolution of the $2 \mathrm{D}$ self-force. It is obvious that the strength of the self-force increases as the vertical sub-bunch size decreases. In addition, the integration for the line charge is split into a singular part and a residual part. The latter is assumed to be weakly dependent on the transverse offset. Therefore, in principle, the sub-bunch method in CSRTrack is not adequate for sub-bunches with a very large transverse-to-longitudinal aspect ratio.

Due to the limit of number of macroparticles in CSRTrack, two million macroparticles out of four million ones from the injector simulation were used. Because of the significant numerical effort, it is difficult to simulate millions of particles in CSRTrack using the sub-bunch model. Considering that the space-charge effects before the slit are negligible, the projected model was used before the slit, while the sub-bunch model using the pseudo-Green's function ( $g_{-} t o \_p$ force in CSRTrack) was employed afterwards. The rms longitudinal sub-bunch size was set to $1 / 20$ of the local rms bunch length and simulation results with three different values for the rms vertical sub-bunch sizes $\left(\sigma_{v, \text { sub }}=\sigma_{v} / 20, \sigma_{v \text {,sub }}=\sigma_{v} / 5\right.$ and $\left.\sigma_{v \text {,sub }}=\sigma_{v}\right)$ were compared, where $\sigma_{v}$ is the local rms vertical bunch size. In the simulation with $\sigma_{v \text {,sub }}=\sigma_{v}$ the bunch almost has the same volume as the original bunch, while a sheetlike bunch was simulated actually in the other two cases. In the following, we will always refer to the results from CSRTrack as being simulated by using $\sigma_{v \text {,sub }}=\sigma_{v}$, unless specified otherwise. In order to avoid a huge vertical-to-longitudinal aspect ratio of the sub-bunch, which was found to introduce unphysical energy modulation in the simulation, the longitudinal subbunch size was not allowed to be smaller than 1/1000 of the vertical sub-bunch size. As a consequence, the longitudinal sub-bunch size will be much larger than $1 / 20$ of the longitudinal bunch size at the end of the compression when the vertical sub-bunch size is large. Moreover, since the code does not allow the horizontal sub-bunch size to be set as a function of the local rms horizontal bunch size, the horizontal rms sub-bunch size was fixed to be $1 / 20$ of the rms horizontal bunch size at the exit of the chicane.

\section{A. Benchmark of the space-charge effects along a drift}

In order to demonstrate the influence of the vertical subbunch size in CSRTrack simulations, the beam dynamics of a 100-MeV, 5-pC cylindrical-symmetric Gaussian bunch was simulated by using IMPACT-T and CSRTrack. The simulations were performed for various initial bunch durations and two initial vertical bunch sizes $(112 \mu \mathrm{m}$ and $28 \mu \mathrm{m})$. The beam was tracked along a $0.1-\mathrm{m}$-long drift space, where the self-force only originates from the space-charge effects.

As shown in Figs. 2 and 3, the results from IMPACT-T and CSRTrack match well except for the 1-fs case in Fig. 2. The study indicates that it is valid to set the vertical sub-bunch size equal to the vertical bunch size in the CSRTrack simulations. If the vertical sub-bunch size is chosen much smaller than the actual vertical size of the beam, the dynamics of a sheetlike bunch is simulated which overestimates the space-charge effects compared to reality. However, if the vertical-to-longitudinal aspect ratio of the bunch is chosen too large (e.g. on the left side of the plots in Fig. 2), in order to avoid a huge vertical-tolongitudinal aspect ratio of the sub-bunch, we were forced to select a big value for the longitudinal sub-bunch size. This choice made the longitudinal sub-bunch size too large to simulate the longitudinal space-charge effect correctly.

\section{PURE MAGNETIC COMPRESSION}

In most magnetic bunch compression systems, the bunch length reaches its minimum at the end of the third dipole magnet. Therefore, the space-charge and CSR effects in the last dipole magnet dominate the emittance growth. However, if the final bunch duration is of the order of sub-fs, the coupling between the transverse and longitudinal phase spaces makes the bunch length at the end of the third dipole magnet still much longer than the final value. For this reason, the impact of the space-charge and 

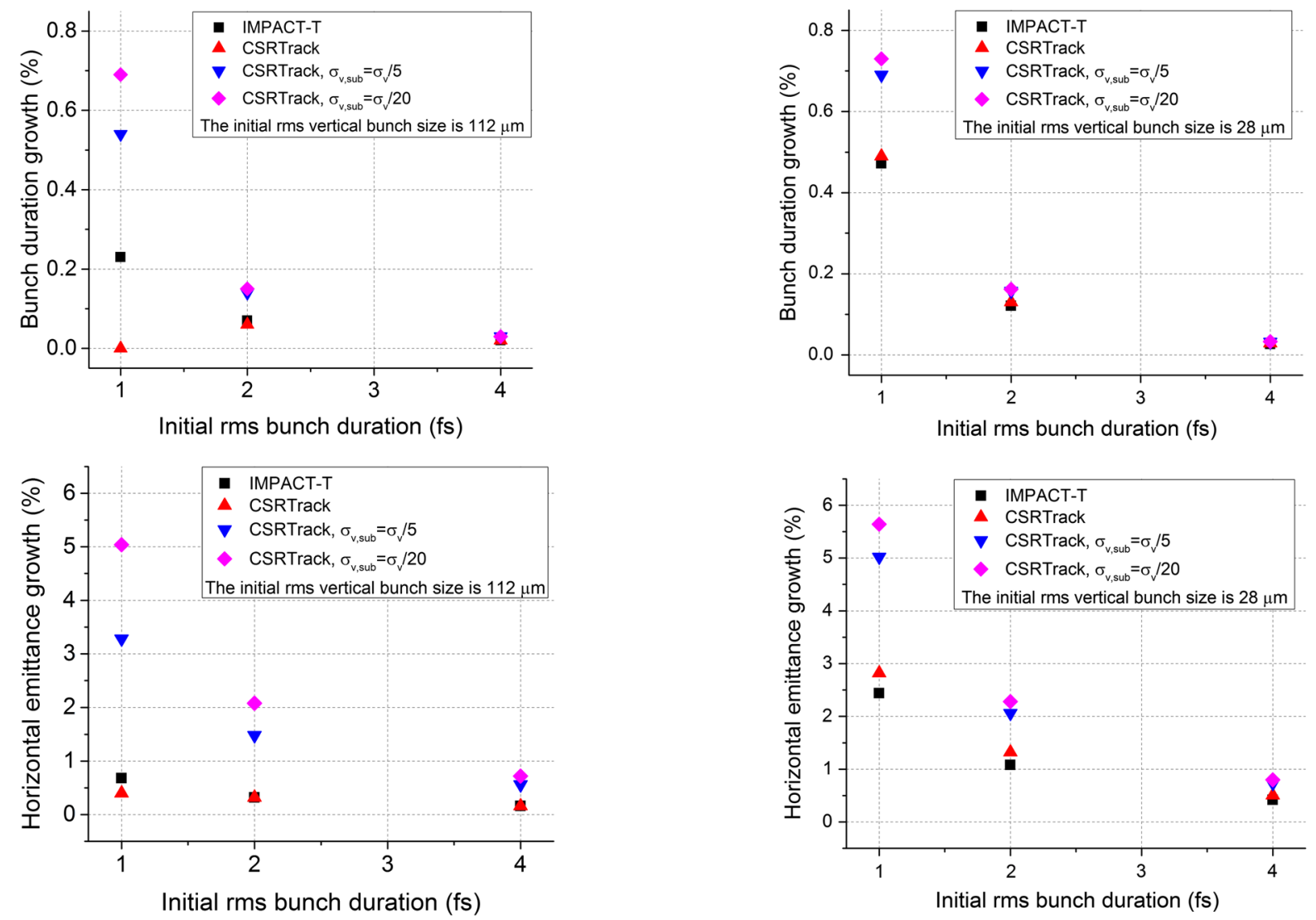

FIG. 2. IMPACT-T and CSRTrack simulation results of the bunch duration and emittance growth of a 100-MeV, 5-pC bunch for different initial bunch durations after drifting after a $0.1 \mathrm{~m}$ long drift. The initial normalized emittance, rms energy spread, rms horizontal and vertical bunch size are $0.25 \mu \mathrm{m}, 0.2 \%, 112 \mu \mathrm{m}$ and $112 \mu \mathrm{m}$ respectively. 50,000 macroparticles were used.

CSR effects is mitigated considerably, which makes it possible to achieve sub-fs electron bunches with relatively high charges via a magnetic bunch compressor.

To first order, taking into account $h R_{56}=-1$ and $R_{56}^{(3)} \approx R_{56}$, the bunch length after the third dipole magnet is approximately given by [22]

$$
\sigma_{z_{3}} \cong \sqrt{R_{56}^{2} \sigma_{\delta_{u}}^{2}+\frac{\varepsilon_{x}}{\beta_{x_{0}}}\left[R_{52}^{(3) 2}+\left(R_{52}^{(3)} \alpha_{x_{0}}-R_{51}^{(3)} \beta_{x_{0}}\right)^{2}\right]},
$$

where $R_{51}{ }^{(3)}, R_{52}{ }^{\left({ }^{3}\right)}, R_{56}{ }^{(3)}$ are the elements of the transfer matrix from the entrance of the chicane to the exit of the third dipole magnet, $\varepsilon_{x}$ is the geometric horizontal emittance of the beam, and $\alpha_{x_{0}}, \beta_{x_{0}}$ are the horizontal CourantSnyder (CS) parameters of the beam at the entrance of the chicane. The contribution of the emittance [the second term on the right-hand side of Eq. (6)] to the bunch length dominates when the final bunch length is very small or the transverse emittance is very large.

\section{A. Simulation results}

Table I summarizes the bunch parameters at the entrance of the chicane for five different bunch charges. The nominal beta functions at the exit of the last dipole magnet are around $10 \mathrm{~m}$ on both planes, while the divergence of the bunch depends on the bunch charge. The full width of the slit is fixed to $0.4 \mathrm{~mm}$ in all cases. The final bunch duration, transverse emittances and energy loss as a function of the final bunch charge are shown in Fig. 4. The final longitudinal phase spaces of the 2.7-pC bunches from different simulations are shown in Fig. 5.

The horizontal emittance increases quickly as the bunch charge increases. The IMPACT-T simulations without CSR effect show that the horizontal emittance growths are no

TABLE I. Summary of bunch parameters at the entrance of the chicane.

\begin{tabular}{lccccc}
\hline \hline Bunch charge (pC) & 10 & 20 & 50 & 100 & 200 \\
rms bunch length (ps) & 2.0 & 2.1 & 2.2 & 2.5 & 2.8 \\
Normalized slice & 0.04 & 0.06 & 0.10 & 0.15 & 0.26 \\
$\quad$ emittance $(\mu \mathrm{m})$ & & & & & \\
Final bunch charge (pC) & 0.4 & 0.7 & 1.6 & 2.7 & 4.8 \\
\hline \hline
\end{tabular}



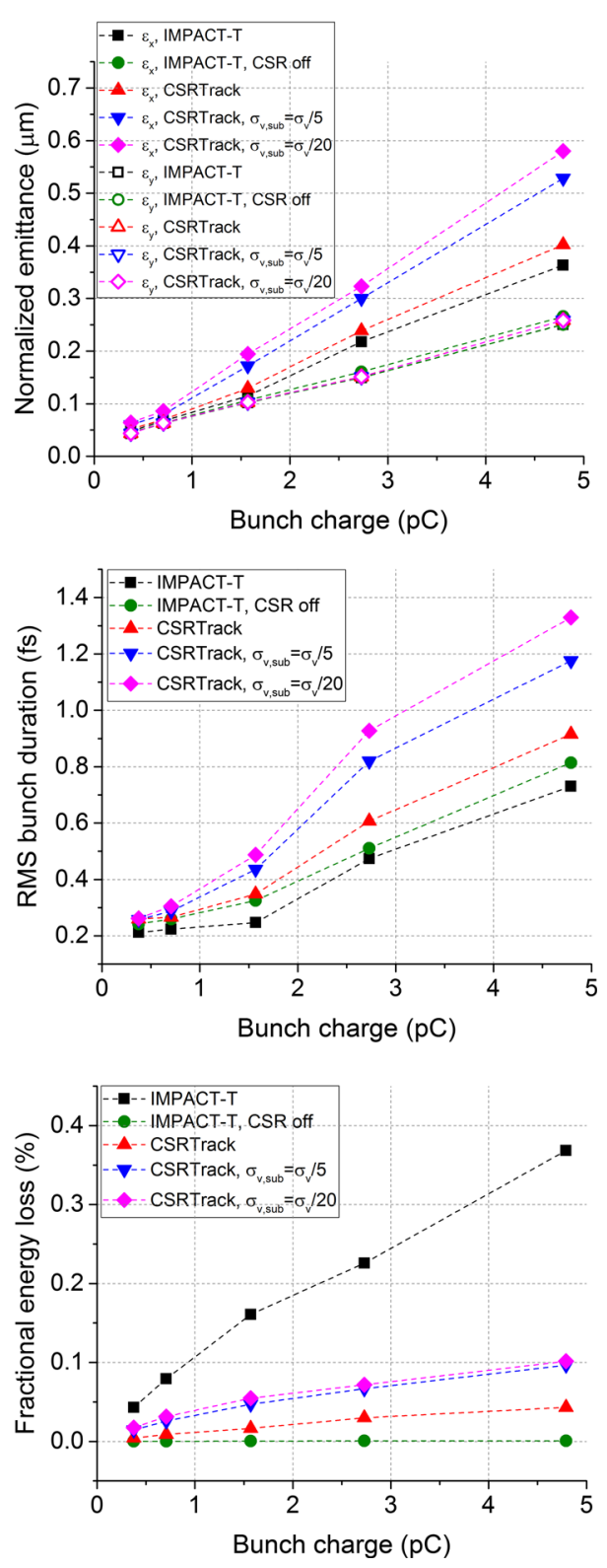

FIG. 4. Comparisons of the bunch durations, normalized emittances and energy losses at the exit of the chicane as simulated by IMPACT-T and CSRTrack. The full width of the slit is $0.4 \mathrm{~mm}$. The initial parameters are summarized in Table I.

more than 5\%. Moreover, according to the benchmark simulations in the previous section, the emittance growth induced by the space-charge effects is expected to be small even for a sheetlike bunch. Therefore, one can conclude that the CSR effect accounts for most of the horizontal emittance dilution. On the other hand, the vertical emittances are preserved in the CSRTrack simulations because of the lack of the vertical self-force, while they increase only slightly in the IMPACT-T simulations since the space-charge effects are not strong.

Although the final longitudinal phase spaces obtained by CSRTrack and IMPACT-T are similar, the bunch durations
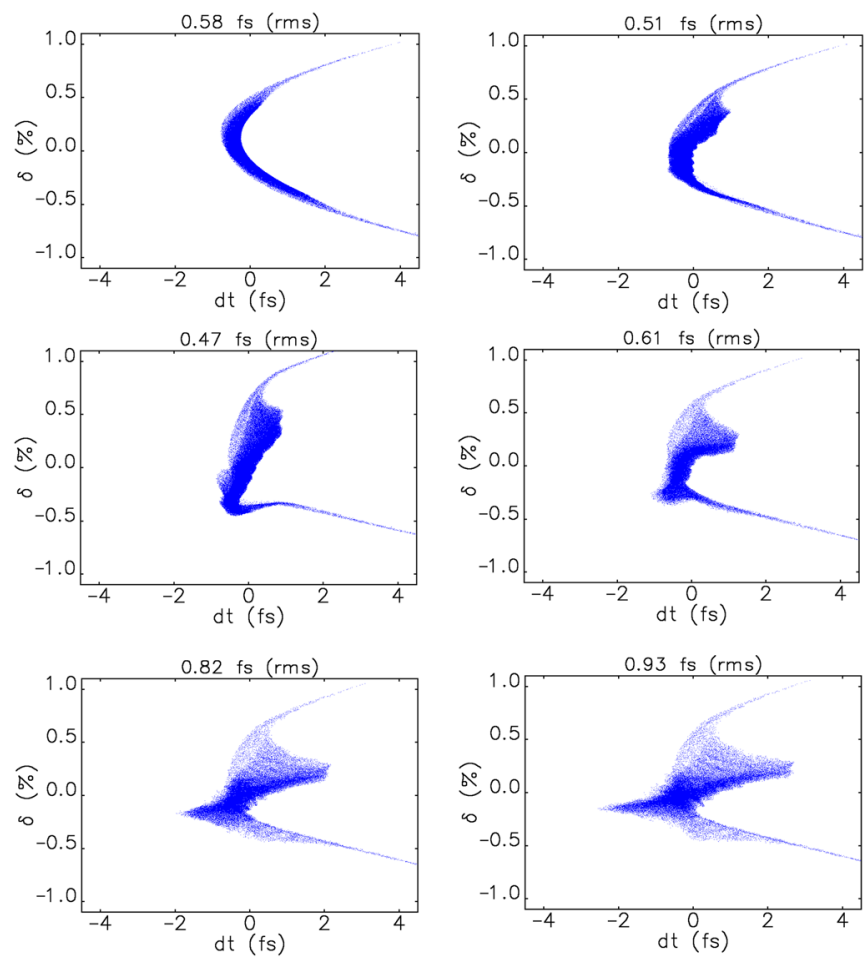

FIG. 5. Final longitudinal phase space simulated by IMPACT-T without self-force (upper left), with only space-charge effects (upper right) and with both space-charge and CSR effects (middle left), as well as simulated by CSRTrack (middle right), with $\sigma_{v \text {,sub }}=\sigma_{v} / 5$ (lower left) and with $\sigma_{v \text {,sub }}=\sigma_{v} / 20$ (lower right). The bunch charge is $2.7 \mathrm{pC}$.

obtained by CSRTrack are $19 \%$ to $41 \%$ longer than those from IMPACT-T, and the horizontal emittances obtained by CSRTrack are up to $13 \%$ higher than those from IMPACT-T. Since the CSR effect dominates, this difference is clearly explained by the different CSR models. In the 1D CSR model, the longitudinal CSR force of the bunch causes the longitudinally dependent energy loss in the dispersion section, which leads to the projected emittance growth. However, since the horizontal CSR force and the horizontal dependence of the longitudinal CSR force are both neglected, the horizontal emittance growth could be underestimated, especially when the bunch has a large horizontal-to-longitudinal aspect ratio. In addition, the results obtained by CSRTrack for a sheetlike bunch indicate that the vertical sub-bunch size also has a significant impact on the calculated CSR effect: the smaller the vertical sub-bunch size, the stronger the CSR effect. According to the algorithm used in CSRTrack, using a smaller vertical sub-bunch size is equivalent to simulating a bunch with a smaller vertical bunch size.

The final bunch duration increases fast as the bunch charge increases. Even if a very narrow slit is used, the second-order effect still dominates the final bunch duration when both the space-charge and CSR effects are not included. It is interesting to find that both the space-charge 

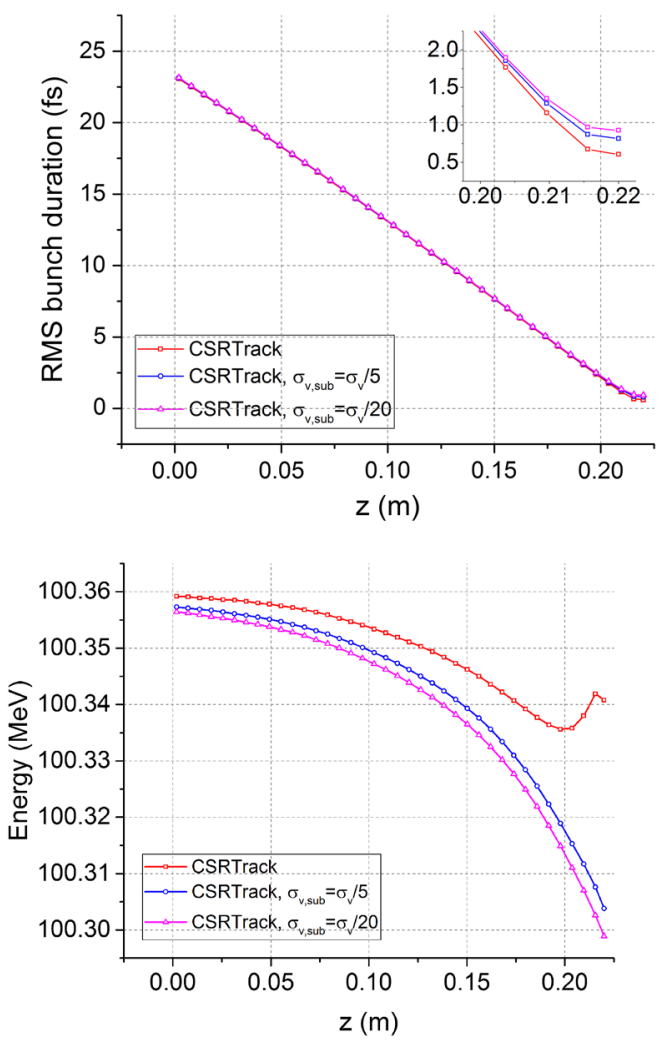

FIG. 6. Evolution of the bunch durations and energies in the last dipole magnet simulated by CSRTrack for different vertical sub-bunch sizes. The bunch charge is $2.7 \mathrm{pC}$.

and CSR effects linearize the longitudinal phase space in the IMPACT-T simulations, which makes the bunch duration even shorter. However, the final bunch duration increases considerably as the CSR effects become stronger, e.g. in the case of the sheetlike bunch results obtained by CSRTrack. The evolution of the bunch duration of the 2.7-pC bunch in the last dipole magnet is shown in Fig. 6. As predicted by Eq. (6), the bunch duration at the entrance is significantly longer than that at the exit.

It is notable that the energy losses obtained by CSRTrack are significantly lower than those obtained by IMPACT-T.

To the largest extent this difference can be attributed to the difference in the geometry of the layouts implemented in the two codes. Due to the presence of the fringe field of the dipole magnet included in IMPACT-T, the exit of the last dipole magnet in the IMPACT-T simulation is about $0.11 \mathrm{~m}$ downstream of the one in the CSRTrack simulation. Strictly speaking, a drift space should be added in the CSRTrack simulation to include the additional energy loss in order to be able to compare the two codes. It should be pointed that the energy loss in the drift space after the last dipole magnet will not affect the bunch emittance and duration because the dispersion is closed.

Another factor that can explain the difference in energy loss between the two codes is that the 1D CSR model is only valid when the dimension of the bunch meets the Derbenev criterion [44], which demands that

$$
\sigma_{\perp} \sigma_{z}^{-2 / 3} \rho^{-1 / 3} \ll 1
$$

where $\rho$ is the bending radius. Rigorous benchmarking simulations with point-to-point L-W solver also prove that the 1D CSR model overestimates the on-axis longitudinal CSR wakefield when the Derbenev criterion is not met [29]. For the beam parameters in our design, the left-hand side of Eq. (7) is larger than 1 in most of the last dipole magnet. Therefore, the 1D CSR model will overestimate the energy loss.

Finally, to a certain extent, the different energy loss can also be attributed to the different final bunch lengths as the CSR power is directly proportional to $\sigma_{z}^{-4 / 3}[16]$.

\section{B. More discussions on the results from CSRTrack}

As discussed in Sec. III, the sub-bunch model implemented in CSRTrack is not adequate for ultrashort bunches with a very large vertical-to-longitudinal aspect ratio. During the bunch compression simulation, it is also found that the energy loss simulated by CSRTrack unphysically changes sign and turns into an energy gain at the end of the last dipole magnet, as shown in Fig. 6. However, this is not the case if the vertical sub-bunch size or the vertical bunch size in the last dipole magnet is small. An example of the former case is the sheetlike bunch results in Fig. 6. For the latter case, we compared the IMPACT-T and CSRTrack simulations with the bunch being more vertically convergent at the entrance of the chicane, so that the vertical bunch size in the last dipole magnet was reduced. The results are shown in Figs. 7 and 8.

On the one hand, the results imply that the abnormal energy change in the CSRTrack simulation does not change the final results abruptly. In fact, since this happens at the end of the last dipole magnet where the dispersion is almost closed, it should have little impact on the final results. On the other hand, these simulations once again show that the vertical bunch size has notable impact on the horizontal emittance and bunch duration. With decreasing vertical bunch size, the 1D CSR model thus increasingly underestimates the horizontal emittance and bunch duration. In addition, the longitudinal phase space distribution in the CSRTrack simulations is affected by the change in the vertical bunch size, while the longitudinal phase space is almost completely unchanged in the IMPACT-T simulations. The results indicate that a large vertical bunch size in the last dipole magnet is required in order to generate a highquality sub-fs electron bunch.

\section{Geometrical wakefields at the slit}

The impact of the transverse wakefield at the slit on the beam emittance was studied analytically. Following [45], 

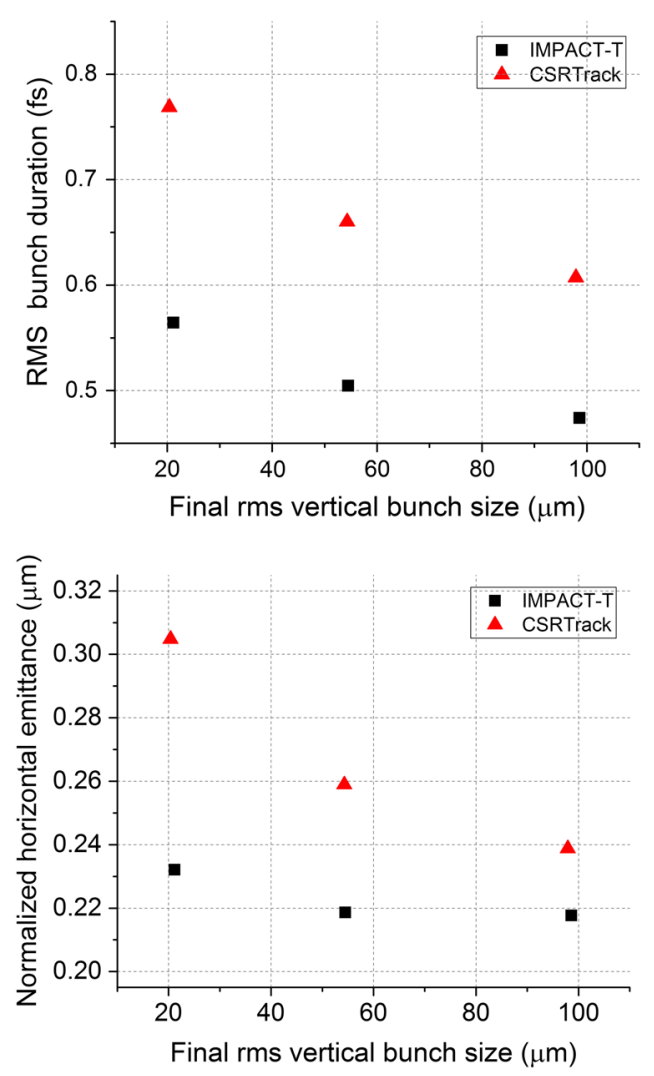

FIG. 7. Final bunch duration and normalized horizontal emittance simulated by IMPACT-T and CSRTrack for different beam optics. The bunch charge is $2.7 \mathrm{pC}$.

the emittance growth was found to be negligible for the typical slit and bunch parameters at the SINBAD linac. This result is in good agreement with the analyses and experiments at FERMI@Elettra FEL [36] and LCLS [37].
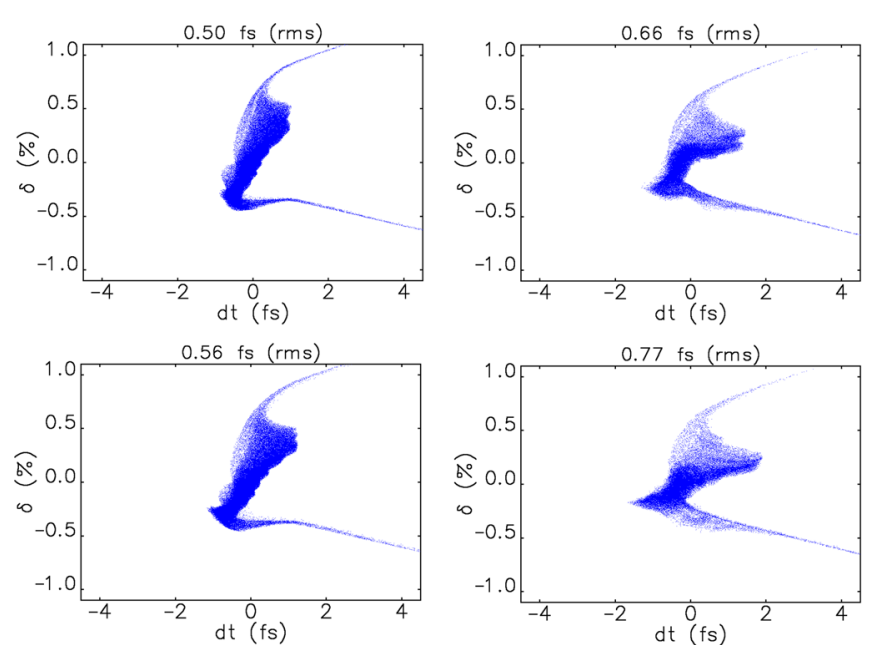

FIG. 8. Final longitudinal phase spaces simulated by IMPACT-T (left column) and CSRTrack (right column). The final vertical bunch sizes are about $54 \mu \mathrm{m}$ (upper row) and $21 \mu \mathrm{m}$ (lower row). The bunch charge is $2.7 \mathrm{pC}$.

\section{HYBRID COMPRESSION}

The major disadvantage of the bunch slicing of highcharge bunches is that a significant amount of charge is lost at the slit, which might cause radiation protection problems. In order to reduce the losses while keeping the same overall performance, a hybrid compression scheme is also proposed. In this scheme, the electron bunch is first compressed significantly by velocity bunching in the linac and then further compressed by the chicane with a slit. As a demonstration, we started the simulation by producing a 10 -pC bunch at the cathode with a 200-fs-long laser pulse. Using the first traveling-wave structure as a rf compressor, the rms bunch length upstream of the chicane is reduced to 183 fs while the shape of the longitudinal phase space is almost linear. The second traveling-wave structure is operated slightly off-crest in order to increase the chirp of the bunch to about $-1 / R_{56}$. By using a 0.3 -mm-wide slit in the chicane, a bunch charge of $2.7 \mathrm{pC}$ can be transported to the exit of the chicane. The bunch durations obtained by IMPACT-T and CSRTrack are both well below $1 \mathrm{fs}$, as shown in Fig. 9. Although the bunch durations are slightly longer than those obtained from pure magnetic compression, the final horizontal emittances become smaller. It is worth noting that only $7.3 \mathrm{pC}$ electrons are lost at the slit, which is more than 1 order of magnitude smaller than the charge lost in the previous case with the same final bunch charge.

Ideally, it is desirable to obtain a bunch short enough at the entrance of the chicane to eliminate the need for a slit. However, when the initial bunch length becomes too short, the CSR effect in the first and second dipole magnets becomes important. The energy losses in the first dipole magnet along a short bunch and along the central slice of a long bunch (the one which survives the transit in the slit) were calculated analytically [16] and are compared in Fig. 10. It is obvious that the energy modulation induced by the CSR effect in a short bunch is significantly higher.

The compression of such short initial bunches was also simulated by IMPACT-T and CSRTrack. For simplicity, the electrons expected to pass through the slit were filtered out from the complete bunch distribution at the entrance of the chicane and tracked afterwards. Since the initial number of
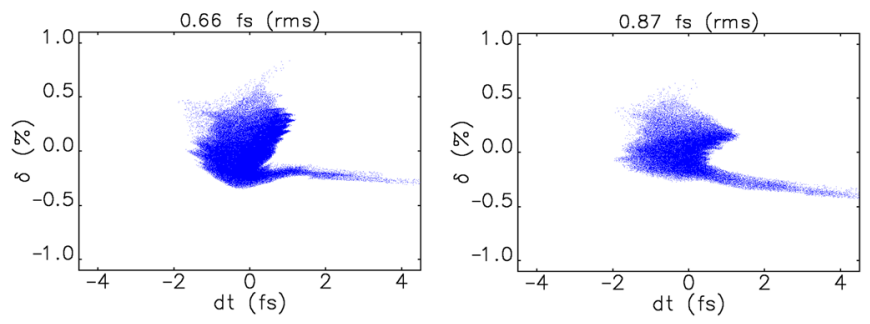

FIG. 9. Final longitudinal phase spaces simulated by IMPACT-T (left) and CSRTrack (right) for the hybrid compression scheme. The final bunch charge is $2.7 \mathrm{pC}$ and the full width of the slit is $0.3 \mathrm{~mm}$. The final normalized horizontal emittances are about 0.19 and $0.21 \mu \mathrm{m}$ respectively. 


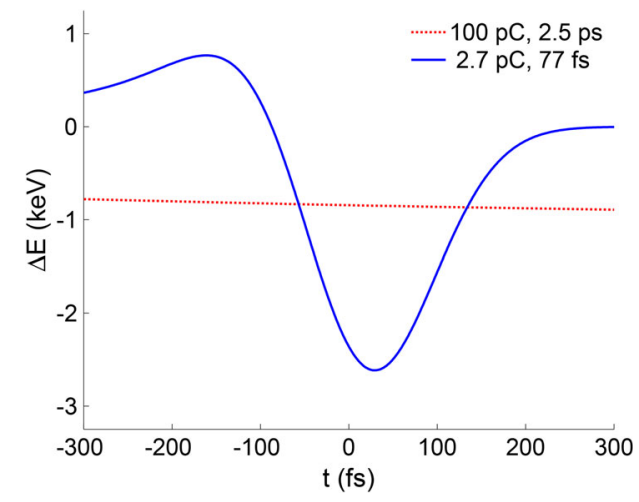

FIG. 10. Analytical results of the energy losses along the bunch in the first dipole magnet for a short bunch compared to the central slice of a long bunch. The steady state CSR model was assumed.

the macroparticles was reduced significantly, the sub-bunch model was employed throughout the complete chicane in the CSRTrack simulations. The typical longitudinal phase space of the central slice of the bunch at the entrance of the chicane and the bunch shape in the middle of the chicane are shown in Fig. 11. The initial portion of the electrons ending up in the surviving slice is only $77 \mathrm{fs}$ long. A comparison of the final bunch durations and horizontal emittances for different bunch charges and the two different codes is shown in Fig. 12, and typical final longitudinal phase spaces of the 2.7-pC bunch are shown in Fig. 13. It is obvious that if the bunch charge is high, the final bunch duration obtained by tracking only the central slice of the bunch is much longer than by starting with the complete bunch. The final longitudinal phase space with the selfforce switched off in the third and fourth dipole magnets was also simulated. It was found that the final bunch becomes longer because of the energy modulation in the first half of the chicane. On the other hand, the CSR effect in the first two dipoles has little impact on the final emittance. It is noteworthy that in the case of tracking only the central slice of a high-charge bunch, the final bunch durations obtained by IMPACT-T and CSRTrack match each other much better than when tracking the complete initial bunches. However, the difference between the final
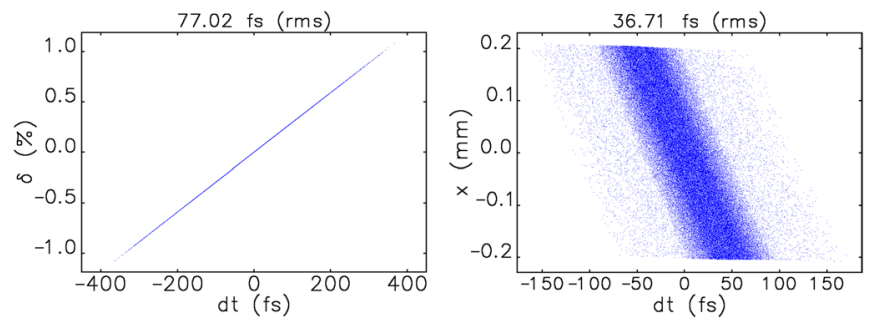

FIG. 11. Typical initial longitudinal phase space (left) and the bunch shape in the middle of the chicane (right) when only the central slice of the bunch was tracked.
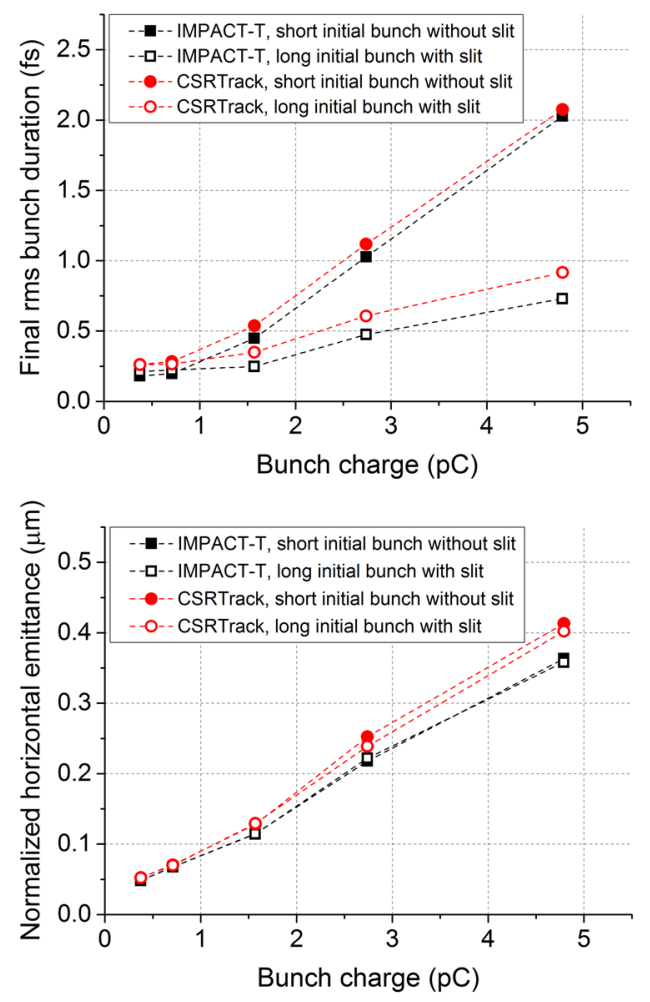

FIG. 12. Comparisons of the final rms bunch durations (up) and horizontal emittances (down) simulated by IMPACT-T and CSRTrack with different initial bunches.

horizontal emittances almost does not change in the two cases.

\section{BUNCH ARRIVAL-TIME JITTER}

In addition to the longitudinal compression of the incoming bunch, a magnetic chicane without a slit also "compresses" the incoming timing jitter and converts the incoming energy jitter into the timing jitter downstream. Both effects are proportional to the compression factor of the bunch [46]. When a slit is placed in the middle of the chicane, the path length of the bunch inside the chicane is well defined by the slit. We will show that the energy jitter upstream of the chicane will be converted into the timing
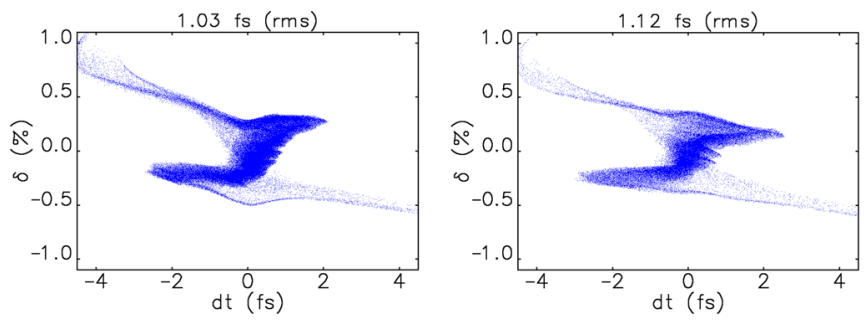

FIG. 13. Final longitudinal phase spaces simulated by IMPACT-T (left) and CSRTrack with $\sigma_{v \text {,sub }}=\sigma_{v}$ (right) by tracking only the central slice of the bunch. The bunch charge is $2.7 \mathrm{pC}$. 
jitter downstream of the chicane in a different way with respect to the case when the slit is not present.

Considering a linac consisting of a gun with output energy $E_{0}$ and $N$ accelerating cavities powered by $N$ independent klystrons, the final energy $E$ can be written as

$$
E=E_{0}+\sum_{i=1}^{N} V_{i} \sin \phi_{i},
$$

where $V_{i}$ and $\phi_{i}=c k_{r f} t_{i}+\phi_{i, 0}$ are the voltage and phase of the $i$ th accelerating cavity respectively, $k_{\mathrm{rf}}$ is the rf wave number, $t_{i}$ is the timing jitter at the entrance of the $i$ th accelerating cavity and $\phi_{i, 0}$ is the reference phase. Here we have assumed that the phase slippage in the accelerating cavities can be neglected, i.e. the velocity bunching is weak. In this case, the timing jitter downstream of the chicane is given by

$$
d t_{b}=d t_{0}+d t_{c}
$$

where $d t_{0}$ is the incoming timing jitter of the whole bunch at the entrance of the linac, $d t_{c}$ is the timing offset jitter of the reference slice with respect to the longitudinal centroid of the whole bunch at the entrance of the chicane. Here the reference slice refers to the slice having the energy of the reference trajectory. Assuming that the longitudinal phase space has a linear chirp $h$, we have

$$
d t_{c} \approx-\frac{1}{h c} \frac{d E}{E},
$$

where

$$
h=e k_{\mathrm{rf}} \sum_{i=1}^{N} \frac{V_{i} \cos \phi_{i}}{E} .
$$

Substituting (10) into (9), one gets

$$
\begin{aligned}
d t_{b} \approx & d t_{0}\left(1-\frac{e k_{r f}}{h E} \sum_{i=1}^{N} V_{i} \cos \phi_{i}\right)+\frac{1}{h c} \frac{d B}{B}-\frac{1}{h c E} d E_{0} \\
& -\frac{e}{h c E} \sum_{i=1}^{N} \sin \phi_{i} d V_{i}-\frac{e}{h c E} \sum_{i=1}^{N} V_{i} \cos \phi_{i} d \phi_{i}
\end{aligned}
$$

where we have used the following relationships:

$$
\begin{aligned}
d E= & d E_{0}-\frac{\partial E}{\partial B} d B+e \sum_{i=1}^{N}\left(\frac{\partial E}{\partial V_{i}} d V_{i}+\frac{\partial E}{\partial \phi_{i}} d \phi_{i}+\frac{\partial E}{\partial t_{0}} d t_{0}\right) \\
= & d E_{0}-\frac{E}{B} d B+e \sum_{i=1}^{N}\left(\sin \phi_{i} d V_{i}+V_{i} \cos \phi_{i} d \phi_{i}\right. \\
& \left.+c k_{r f} V_{i} \cos \phi_{i} d t_{0}\right) .
\end{aligned}
$$

The magnetic field jitter is included in Eq. (13) because the energy of the reference trajectory is proportional to the magnetic field of the dipole magnets. Substituting Eq. (11) into Eq. (12), the first term on the right-hand side of Eq. (12) vanishes. Finally, the rms timing jitter can be written as

$$
\sigma_{t_{b}} \approx \frac{1}{c} \sqrt{\left(\frac{e}{h E}\right)^{2} \sum_{i=1}^{N} V_{i}^{2}\left(\sin ^{2} \phi_{i} \sigma_{\delta_{V_{i}}}^{2}+\cos ^{2} \phi_{i} \sigma_{\phi_{i}}^{2}\right)},
$$

where $\delta_{E_{0}}=d E_{0} / E_{0}, \delta_{V_{i}}=d V_{i} / V_{i}, \delta_{\phi_{i}}=d \phi_{i} / \phi_{i}$ and $\delta_{B}=d B / B$. Considering a beam with certain energy and chirp, and assuming that the rms jitters of each accelerating cavity are the same, Eq. (14) reaches its minimum when all the cavities have the same voltage and phase. Therefore, the minimum timing jitter downstream of the chicane is given by

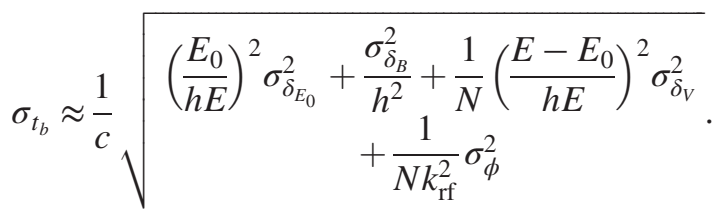

It is obvious that, regardless of the compression factor of the bunch, the incoming timing jitter will be fully "compressed" while the phase jitter of the accelerating cavity will be fully converted into the timing jitter downstream. At the SINBAD linac, taking into account the timing jitter introduced by the gun charge jitter observed in the simulations [42], the timing jitter downstream of the chicane for the pure magnetic compression case is then given by

$$
\sigma_{t_{b}} \approx \frac{1}{c} \sqrt{\begin{array}{c}
R_{56}^{2}\left[\frac{E_{0}^{2}}{E^{2}} \sigma_{\delta_{E_{0}}}^{2}+\sigma_{\delta_{B}}^{2}+\frac{1}{2}\left(1-\frac{E_{0}}{E}\right)^{2} \sigma_{\delta_{V}}^{2}\right] \\
+\frac{1}{2 k_{\mathrm{rf}}^{2}} \sigma_{\phi}^{2}+D \sigma_{\delta_{Q}}^{2}
\end{array},}
$$

where $\delta_{Q}=d Q / Q$ and $D$ is a coefficient depending on the bunch charge. One possible explanation for the charge jitter term is that the charge jitter could introduce perturbation into the longitudinal phase space shape due to the space-charge effects, which will in turn affect the longitudinal position of the reference slice. The gun energy jitter only slightly increases the timing jitter, e.g. a gun energy jitter of $0.1 \%$ corresponds to a timing jitter of $1.7 \mathrm{fs}$.

The timing jitters downstream of the chicane for different bunch charges and compression schemes were studied by S2E simulations. Since IMPACT-T is not suitable for timing jitter studies, ELEGANT [47] was used instead to simulate the beam dynamics downstream of the traveling-wave structure. The sensitivity of each jitter sources was first studied individually, i.e. the particles were tracked by assigning an artificial jitter to one of the sources while 
TABLE II. Summary of jitter sensitivity and tolerance studies.

\begin{tabular}{|c|c|c|c|c|c|c|c|}
\hline \multirow[b]{2}{*}{ Jitter source } & \multirow[b]{2}{*}{ Unit } & \multicolumn{3}{|c|}{ Sensitivity for $10-$ fs timing jitter } & \multicolumn{3}{|c|}{ rms tolerance } \\
\hline & & $20 \mathrm{pC}$ & $100 \mathrm{pC}$ & $10 \mathrm{pC}^{\text {hybrid }}$ & $20 \mathrm{pC}$ & $100 \mathrm{pC}$ & $10 \mathrm{pC}^{\text {hybrid }}$ \\
\hline Laser-to-rf & fs & 42437.1 & 5949.7 & 159.8 & 200.0 & 200.0 & 50.0 \\
\hline Gun charge & $\%$ & 5.8 & 1.6 & 301.6 & 1.0 & $0.3(1.0)$ & 4.0 \\
\hline Gun phase & $\operatorname{deg}$ & 1.75 & 0.78 & 0.61 & 0.06 & 0.06 & 0.06 \\
\hline Gun amplitude & $\%$ & 0.61 & 1.14 & 0.72 & 0.06 & 0.06 & 0.06 \\
\hline Traveling-wave structure 1 phase & $\operatorname{deg}$ & 0.021 & 0.021 & 0.011 & 0.013 & $0.013(0.010)$ & 0.009 \\
\hline Traveling-wave structure 2 phase & deg & 0.022 & 0.022 & 0.13 & 0.013 & $0.013(0.010)$ & 0.011 \\
\hline Traveling-wave structure 1 amplitude & $\%$ & 0.055 & 0.055 & 0.073 & 0.013 & $0.013(0.010)$ & 0.009 \\
\hline Traveling-wave structure 2 amplitude & $\%$ & 0.064 & 0.064 & 0.040 & 0.013 & $0.013(0.010)$ & 0.011 \\
\hline Magnetic field & $\%$ & 0.030 & 0.030 & 0.030 & 0.01 & 0.01 & 0.01 \\
\hline Total rms timing jitter & fs & $\ldots$ & $\cdots$ & $\ldots$ & 9.98 & $10.13(10.49)$ & 9.72 \\
\hline
\end{tabular}

assuming no additional jitters from the other sources. Within the studied ranges, the timing jitter was found to be a linear function of the jitter amplitude for each of the jitter sources. Once the sensitivities of all the jitter sources are known, the expected rms timing jitter for a certain tolerance budget is approximately given by

$$
\sigma_{t_{b}} \approx 10 \sqrt{\sum_{i=1}^{n}\left(\frac{j_{\mathrm{tol}, i}}{j_{\mathrm{sen}, i}}\right)^{2}}[\mathrm{fs}]
$$

where $j_{\text {sen, } i}$ refers to the amplitude of the $i$ th jitter source corresponding to a 10 -fs timing jitter and $j_{\text {tol }, i}$ is the rms tolerance of the $i$ th jitter source. Equation (17) can be used to quickly estimate a tolerance budget for a timing jitter of less than $10 \mathrm{fs}$. The statistic total rms timing jitter with the tolerance budget was calculated after 300 randomized S2E simulations, where a 3-sigma Gaussian distribution for each jitter source was assumed. The simulation results are summarized in Table II.

In the pure magnetic compression case, the tolerances for the rf phase and amplitude jitters are demanding, being 0.013 degree and $0.013 \%$ respectively. The charge jitter is required to be $1 \%$ for the $20 \mathrm{pC}$ case, and as low as $0.3 \%$ for the $100 \mathrm{pC}$ case in order to keep the same stability requirements for the traveling-wave structure. Alternatively, if the charge jitter can only be limited to $1 \%$, the tolerance on the phase and amplitude has to be reduced to 0.01 degree and $0.01 \%$ respectively.

In the hybrid compression case, the tolerances of the traveling-wave structure are even more demanding. Especially, the phase and amplitude tolerances of the first traveling-wave structure are as small as 0.009 degree and $0.009 \%$ respectively. However, the bunch charge jitter in the hybrid compression case is negligible.

The transverse position jitter of the beam is an additional potential source of the final charge jitter. Simulations have shown that the final rms charge jitter is less than $0.3 \%$ by assuming a rms horizontal position jitter of $10 \mu \mathrm{m}$ at the slit and thus can be neglected.
In summary, the tolerance budget of the traveling-wave structure for the sub-10-fs timing jitter is very challenging but not unreasonable. At REGAE (Relativistic Electron Gun for Atomic Exploration) at DESY, the amplitude and phase jitter of the S-band gun is expected to be $0.01 \%$ and 0.01 degree respectively [48], and a similar technology is foreseen to be implemented at the SINBAD facility. At the SwissFEL Injector Test Facility, a shot-to-shot rms stability of $0.02 \mathrm{deg}$ for the phase and 0.02 for the amplitude has been achieved at the $S$-band system [49].

\section{CONCLUSIONS}

In this paper, we have reported simulation studies of the generation of $100 \mathrm{MeV}$, sub-fs electron bunches with sub10 -fs bunch arrival-time jitter (timing jitter) at the SINBAD linac. A weak magnetic chicane $\left(R_{56} \approx-10 \mathrm{~mm}\right)$ with a sub-mm-wide slit (collimator) between the second and the third dipole magnets is used to fully compress the central slice of an incoming long bunch. Because of the slit, the bunch length in the first half of the chicane can be long enough to ensure a negligible energy modulation induced by the CSR effect. This feature is of vital importance in generating sub-fs bunches with high bunch charges.

The beam dynamics inside the chicane was studied by using IMPACT-T and CSRTrack with different space-charge and CSR models. Due to the coupling between the horizontal and longitudinal phase space, the bunch duration at the entrance of the last dipole magnet is still much longer than the final value. This fact mitigates considerably the impact of the space-charge and CSR effects on the beam quality. Simulations from both codes proved that sub-fs electron bunches with charges up to $4.8 \mathrm{pC}$ are achievable. It is found that the CSR effect dominates the horizontal emittance growth, and the different CSR models account mostly for the difference between the results obtained by the two codes. Compared to the 3D CSR model in CSRTrack, the 1D CSR model in IMPACT-T underestimates the final bunch duration and emittance when the transverse-tolongitudinal aspect ratio of the bunch is very large. 
Particularly, the CSR effect is also strongly affected by the vertical bunch size. Our studies also show that in CSRTrack simulations it is important to set the vertical sub-bunch size equal to the vertical bunch size in order not to overestimate the space-charge and CSR effects. When the vertical subbunch size is set to a value much smaller than the actual vertical bunch size, the beam dynamics of a sheetlike bunch will be simulated actually, which could lead to an incorrect result.

In order to achieve a bunch arrival-time jitter of less than $10 \mathrm{fs}$, it is of vital importance to use a weak chicane, and the stabilization of the phase and amplitude jitter of the traveling-wave structures is critical and challenging. These requirements can be relaxed to 0.013 degree and $0.013 \%$ stability respectively by driving each of the traveling-wave structures with independent klystrons. When operating with high charge bunches, the extracted charge jitter at the photocathode or the phase and amplitude of the traveling-wave structures need to be further stabilized.

A two-stage hybrid compression scheme is also proposed in order to reduce the charge loss at the slit in the high-charge cases. Simulations show that the final bunch duration becomes slightly longer while the horizontal emittance dilution is mitigated with respect to the pure magnetic compression case. Since the two traveling-wave structures are operated at different phases and amplitudes, the stabilization of the traveling-wave structures is even more challenging than in the pure magnetic compression case.

\section{ACKNOWLEDGMENTS}

The authors would like to thank R. Brinkmann for his support and acknowledge H. Schlarb and K. Floettmann for their extensive advices on the tolerance budget of synchronization.

[1] T. Tajima and J. M. Dawson, Laser Electron Accelerator, Phys. Rev. Lett. 43, 267 (1979).

[2] J. Faure, Y. Glinec, A. Pukhov, S. Kiselev, S. Gordienko, E. Lefebvre, J.-P. Rousseau, F. Burgy, and V. Malka, A laser-plasma accelerator producing monoenergetic electron beams, Nature (London) 431, 541 (2004).

[3] W. P. Leemans, B. Nagler, A. J. Gonsalves, Cs. Tóth, K. Nakamura, C. G. R. Geddes, E. Esarey, C. B. Schroeder, and S. M. Hooker, GeV electron beams from a centimetrescale accelerator, Nat. Phys. 2, 696 (2006).

[4] E. Guillaume, A. Döpp, C. Thaury, A. Lifschitz, J.-P. Goddet, A. Tafzi, F. Sylla, G. Iaquanello, T. Lefrou, P. Rousseau, K. TaPhuoc, and V. Malka, Physics of fullyloaded laser-plasma accelerators, Phys. Rev. ST Accel. Beams 18, 061301 (2015).

[5] J. Grebenyuk, R. Assmann, U. Dorda et al., Laser-driven acceleration with external injection at SINBAD, in Proceedings of IPAC'14 (JACoW, Dresden, Germany, 2014), TUPME064.
[6] I. Blumenfeld, C. E. Clayton, F. J. Decker et al., Energy doubling of $42 \mathrm{GeV}$ electrons in a metre-scale plasma wakefield accelerator, Nature (London) 445, 741 (2007).

[7] F. Krausz and M. Ivanov, Attosecond physics, Rev. Mod. Phys. 81, 163 (2009).

[8] L. Gallmann, C. Cirelli, and U. Keller, Attosecond science: Recent highlights and future trends, Annu. Rev. Phys. Chem. 63, 447 (2012).

[9] J. Rosenzweig, D. Alesini, G. Andonian et al., Generation of ultra-short, high brightness electron beams for singlespike SASE FEL operation, Nucl. Instrum. Methods Phys. Res., Sect. A 593, 39 (2008).

[10] B. Marchetti, R. W. Assmann, C. Behrens et al., Ares: Accelerator research experiment at SINBAD, in Proceedings of IPAC15 (JACoW, Richmond, VA, 2015), TUPWA029.

[11] R. Assmann, C. Behrens, R. Brinkmann et al., SINBAD-A proposal for a dedicated accelerator research facility at DESY, in Proceedings of IPAC'14 (JACoW, Dresden, Germany, 2014), TUPME047.

[12] A. Bacci and A. R. Rossi, Ultra-short electron bunches by Velocity Bunching as required for plasma wave accelerations, Nucl. Instrum. Methods Phys. Res., Sect. A 740, 42 (2014).

[13] K. Kan, J. Yang, T. Kondoh, K. Norizawa, A. Ogata, T. Kozawa, and Y. Yoshida, Simulation study of subfemtosecond electron bunch generation using photocathode RF gun linac, Nucl. Instrum. Methods Phys. Res., Sect. A 622, 35 (2010).

[14] M. Ferrario, D. Alesini, A. Bacci et al., Experimental Demonstration of Emittance Compensation with Velocity Bunching, Phys. Rev. Lett. 104, 054801 (2010).

[15] R. Talman, Novel Relativistic Effect Important in Accelerators, Phys. Rev. Lett. 56, 1429 (1986).

[16] E. L. Saldin, E. A. Schneidmiller, and M. V. Yurkov, On the coherent radiation of an electron bunch moving in an arc of a circle, Nucl. Instrum. Methods Phys. Res., Sect. A 398, 373 (1997).

[17] H. H. Braun, R. Corsini, L. Groening, F. Zhou, A. Kabel, T. O. Raubenheimer, R. Li, and T. Limberg, Emittance growth and energy loss due to coherent synchrotron radiation in a bunch compressor, Phys. Rev. ST Accel. Beams 3, 124402 (2000).

[18] M. Dohlus, T. Limberg, and P. Emma, Electron bunch length compression, ICFA Beam Dynamics Newsletter 38, 39 (2005).

[19] C. Mitchell, J. Qiang, and P. Emma, Longitudinal pulse shaping for the suppression of coherent synchrotron radiation-induced emittance growth, Phys. Rev. ST Accel. Beams 16, 060703 (2013).

[20] C. C. Hall, S. G. Biedron, A. L. Edelen, S. V. Milton, S. Benson, D. Douglas, R. Li, C. D. Tennant, and B. E. Carlsten, Measurement and simulation of the impact of coherent synchrotron radiation on the Jefferson Laboratory energy recovery linac electron beam, Phys. Rev. ST Accel. Beams 18, 030706 (2015).

[21] C. Prokop, P. Piot, B. E. Carlsten, and M. Church, Beam dynamics performances and applications of a low-energy electron-beam magnetic bunch compressor, Nucl. Instrum. Methods Phys. Res., Sect. A 719, 17 (2013). 
[22] J. Zhu, P. Piot, D. Mihalcea, and C. R. Prokop, Formation of compressed flat electron beams with high transverseemittance ratios, Phys. Rev. ST Accel. Beams 17, 084401 (2014).

[23] M. Schreck and P. Wesolowski, Analytical bunch compression studies for a linac-based electron accelerator, Phys. Rev. ST Accel. Beams 18, 100101 (2015).

[24] A. He, F. Willeke, L. H. Yu, L. Yang, T. Shaftan, G. Wang, Y. Li, Y. Hidaka, and J. Qiang, Design of low energy bunch compressors with space charge effects, Phys. Rev. ST Accel. Beams 18, 014201 (2015).

[25] B. E. C. Koltenbah, C. G. Parazzoli, R. B. Greegor, and D. H. Dowell, PARMELA_B: A new version of PARMELA with coherent synchrotron radiation effects and a finite difference space charge routine, Nucl. Instrum. Methods Phys. Res., Sect. A 487, 249 (2002).

[26] J. Qiang, S. Lidia, R. D. Ryne, and C. Limborg-Deprey, Three-dimensional quasistatic model for high brightness beam dynamics simulation, Phys. Rev. ST Accel. Beams 9 , 044204 (2006).

[27] J. Qiang, C. Mitchell, and R. Ryne, A fast high-order method to calculate wakefields in an electron beam, Nucl. Instrum. Methods Phys. Res., Sect. A 682, 49 (2012).

[28] http://amac.lbl.gov/ jiqiang/impact/.

[29] R. D. Ryne et al., Large scale simulation of synchrotron radiation using a Lienard-Wiechert approach, in Proceedings of the 3rd International Particle Accelerator Conference, New Orleans, LA, 2012 (IEEE, Piscataway, NJ, 2012).

[30] M. Dohlus et al., CSRtrack: Faster calculation of 3-D CSR effects, in Proceedings of the 2004 FEL Conference (Comitato Conferenze Elettra, Trieste, Italy, 2004), pp. 18-21.

[31] http://www-mpy.desy.de/trafic4/TraFiC4/.

[32] L. Giannessi and M. Quattromini, TREDI simulations for high-brilliance photoinjectors and magnetic chicanes, Phys. Rev. ST Accel. Beams 6, 120101 (2003).

[33] R. D. Ryne, C. E. Mitchell, J. Qiang, and B. Carlsten, Using a Lienard-Wiechert solver to study coherent synchrotron radiation effects, in Proceedings of FEL2013 (JACoW, New York, NY, 2013), MOOCNO04.

[34] M. Borland, Potential production of ultrashort electron bunches with the Advanced Photon Source linac, in Proceedings of the 19th Particle Accelerator Conference, Chicago, IL, 2001 (IEEE, Piscataway, NJ, 2001).

[35] P. Emma, K. Bane, M. Cornacchia, Z. Huang, H. Schlarb, G. Stupakov, and D. Walz, Femtosecond and Subfemtosecond X-Ray Pulses from a Self-Amplified SpontaneousEmission-Based Free-Electron Laser, Phys. Rev. Lett. 92, 074801 (2004).
[36] S. Di Mitri, D. Castronovo, I. Cudin, and L. Froehlich, Electron slicing for the generation of tunable femtosecond soft X-ray pulses from a free electron laser and slice diagnostics, Phys. Rev. ST Accel. Beams 16, 042801 (2013).

[37] F. Zhou, K. Bane, Y. Ding, Z. Huang, H. Loos, and T. Raubenheimer, Measurements and analysis of a highbrightness electron beam collimated in a magnetic bunch compressor, Phys. Rev. ST Accel. Beams 18, 050702 (2015).

[38] K. Floettmann, T. Limberg, and P. Piot, Report No. TESLAFEL-2001-06, DESY, Hamburg, 2001.

[39] R. Assmann and K. Yokoya, Transverse beam dynamics in plasma-based linacs, Nucl. Instrum. Methods Phys. Res., Sect. A 410, 544 (1998).

[40] T. O. Raubenheimer and F. Zimmermann, Final-focus systems in linear colliders, Rev. Mod. Phys. 72, 95 (2000).

[41] K. Floettmann, ASTRA particle tracking code http://tesla .desy.de/ meykopff/.

[42] J. Zhu et al., Timing jitter studies for sub-fs electron bunch generation at SINBAD, in Proceedings of IPAC15 (JACoW, Richmond, VA, 2015), WEPMA031.

[43] M. Dohlus, A. Kabel, and T. Limberg, Efficient field calculation of 3D bunches on general trajectories, Nucl. Instrum. Methods Phys. Res., Sect. A 445, 338 (2000); M. Dohlus, Report No. TESLA-FEL-2003-05.

[44] Y.S. Derbenev, J. Rossbach, E. L. Saldin, and V. D. Shiltsev, Report No. TESLA-FEL 95-05.

[45] P. Tenenbaum, K. L. F. Bane, L. Eriksson et al., Direct measurement of the transverse wakefields of tapered collimators, Phys. Rev. ST Accel. Beams 10, 034401 (2007).

[46] P. Craievich, S. Di Mitri, M. Milloch, G. Penco, and F. Rossi, Modeling and experimental study to identify arrivaltime jitter sources in the presence of a magnetic chicane, Phys. Rev. ST Accel. Beams 16, 090401 (2013).

[47] M. Borland, ELEGANT: A Flexible SDDS-Compliant Code for Accelerator Simulation, Advanced Photon Source LS-287, 2000.

[48] M. Felber, M. Hoffmann, U. Mavric et al., Laser synchronization at regae using phase detection at an intermediate frequency, in Proceedings of the 3rd International Particle Accelerator Conference, New Orleans, LA, 2012 (Ref. [29]), WEPPD048.

[49] B. Beutner and S. Reiche, Sensitivity and tolerance study for the SwissFEL, in Proceedings of the 32nd Free Electron Laser Conference, Malmö, Sweden (Max-lab, Sweden, 2010), WEPB17. 